Title: Deletion of the voltage-gated calcium channel, Cav1.3, causes deficits in motor performance and associative learning

Authors: Lauffer, Marisol ${ }^{1}$; Wen, Hsiang ${ }^{1,2,3}$; Myers, Bryn ${ }^{3}$; Plumb, Ashley ${ }^{4}$; Parker, Krystal $^{1,2,3}$; Williams, Aislinn ${ }^{1,2,3}$

Affiliations: ${ }^{1}$ lowa Neuroscience Institute, ${ }^{2}$ Department of Psychiatry, ${ }^{3}$ Carver College of Medicine, ${ }^{4}$ Department of Physical Therapy and Rehabilitation Science, University of lowa, lowa City, IA 52242, USA

Correspondence:

Aislinn Williams

University of lowa

169 Newton Road

2326 PBDB

lowa City, IA 52242

Email: aislinn-williams@uiowa.edu

Telephone: 319-335-2434

Fax: 319-335-3349 
Abstract

L-type voltage-gated calcium channels (LVGCCs) are important regulators of neuronal activity and are widely expressed throughout the brain. One of the major LVGCC isoforms in the brain is Cav1.3. Mice lacking Cav1.3 (Cav1.3 KO) have impairments in fear conditioning and depressive-like behaviors, which have been linked to the role of Cav1.3 in hippocampal and amygdala function. Genetic variation in Cav1.3 has been linked to a variety of psychiatric disorders, including autism and schizophrenia, which are associated with motor, learning, and social deficits. Here, we explored whether Cav1.3 plays a role in these behaviors. We found that Cav1.3 KO mice have deficits in rotarod learning despite normal locomotor function. Deletion of Cav1.3 is also associated with impaired associative learning on the Erasmus Ladder. We did not observe any impairments in Cav1.3 KO mice on assays of anxiety-like, depression-like, or social preference behaviors. Our results suggest an important role for Cav1.3 in neural circuits involved in motor learning and concur with previous data showing its involvement in associative learning.

Keywords: calcium channels, knockout mouse, learning, motor activity, cerebellum, behavioral genetics 
Introduction

Genome-wide association studies have identified many loci relevant to the risk for neuropsychiatric disorders such as autism, schizophrenia, and bipolar disorder, but the mechanisms by which these genes modify risk is unclear in most cases. One group of genes robustly linked to neuropsychiatric disease are the L-type voltage-gated calcium channel (LVGCC) genes, including CACNA1D. The CACNA1D gene encodes the pore-forming subunit of the L-type voltage-gated calcium channel, Cav1.3, which is expressed in many tissues including the brain, heart, inner ear, and adrenal glands (Catterall, Perez-Reyes, Snutch, \& Striessnig, 2005). The CACNA1D gene has been linked to several neuropsychiatric disorders, including autism, bipolar disorder, depression, schizophrenia, and Parkinson's disease (Ament et al., 2015; CrossDisorder Group of the Psychiatric Genomics, 2013; Pinggera et al., 2015; Psychiatric, 2011; Scholl et al., 2013). Although the genetic connection between CACNA1D and neuropsychiatric disease is well-established, the functional role(s) of Cav1.3 in different neural circuits remains under active investigation.

Loss of Cav1.3 has been associated with multiple functional deficits in nervous system development and function. Due to its essential role in inner ear development (Brandt, Striessnig, \& Moser, 2003), Cav1.3 knockout mice are congenitally deaf (Platzer et al., 2000), and therefore cannot undergo standard hearing-dependent associative learning paradigms, such as standard tone-paired fear conditioning (McKinney \& Murphy, 2006). In contrast, although Cav1.3 influences light 
responsiveness in the retina (Busquet et al., 2010; Shi, Chang, Yu, Ko, \& Ko, 2017), mice lacking Cav1.3 exhibit normal performance on vision-dependent tasks, such as the visible platform version of the Morris water maze (Busquet et al., 2010; McKinney \& Murphy, 2006). Cav1.3 is expressed in the hippocampus, and Cav1.3 KO mice have impaired object location memory, which is hippocampus-dependent (Kim et al., 2017; Marschallinger et al., 2015), However, Cav1.3 KO mice have normal performance on the hidden platform version of the Morris water maze (McKinney \& Murphy, 2006) and novel object recognition (Kim et al., 2017) which are both mediated by the hippocampus. Some groups have identified abnormalities in anxiety- and depressionlike behaviors in Cav1.3 KO mice (Busquet et al., 2010), although not all groups have found similar deficits (Marschallinger et al., 2015; McKinney \& Murphy, 2006). Pharmacological inhibition of Cav1.3 in Cav1.2 dihydropyridine insensitive mutant mice specifically in the ventral tegmental area caused no abnormalities in anxiety-like, depression-like, or social behaviors (Martinez-Rivera et al., 2017).

Although CACNA1D is expressed in the cerebellum (Hell et al., 1993), the specific role of Cav1.3 in motor learning circuits has been relatively unexplored. Previous studies in $\mathrm{Cav}_{\mathrm{v}} 1.3 \mathrm{KO}$ mice have not shown deficits in locomotor function on fixed-speed rotarod (Clark et al., 2003) or swim speed (Busquet et al., 2010; McKinney \& Murphy, 2006), but one study using accelerating rotarod in a small sample showed a trend toward impaired motor learning over time (McKinney \& Murphy, 2006). We hypothesized that larger sample sizes and different tasks might reveal deficits in motor function and learning in Cav1.3 KO mice. Here we explored the role of Cav1.3 in motor, learning, and social behaviors, and found that Cav1.3 $\mathrm{KO}$ mice have impairments in 
rotarod learning despite intact baseline locomotor function. We also find that Cav1.3 KO mice display impaired associative learning on the Erasmus Ladder task without evidence of ataxia or motor incoordination. We find no deficits in Cav1.3 KO mice in affective-like, anxiety-like, or social behaviors. Our results suggest that Cav1.3 plays an important role in the neural circuits essential for motor and associative learning. Given its importance in shaping neuronal activity and behavior in the hippocampus and amygdala, we hypothesized that loss of Cav1.3 would cause abnormalities in cerebellarmediated motor learning.

Materials and Methods

Mice

The generation of $\mathrm{Ca}_{v} 1.3$ knockout $(\mathrm{KO})$ mice has been described previously (Platzer et al., 2000). Breeding pairs of $\mathrm{Ca}_{v} 1.3$ haploinsufficient (Hap) mice on a C57BL/6N background were maintained on a C57BL/6N background by crossing Hap offspring with C57BL/6N WT mice purchased from Charles River (Wilmington, MA). For all experiments, male and female $\mathrm{Ca}_{v} 1.3 \mathrm{Hap}$ mice, $\mathrm{KO}$ mice, and their wild-type littermates (WT) were used. All mice were adults (10-33 weeks old) at the time of testing. Behavioral experiments were run with two independent cohorts of mice in the following order: cohort 1 underwent Erasmus Ladder, rotarod, forced swim, and tail suspension; cohort 2 underwent elevated zero maze, open field, 3-chamber social preference test, rotarod, tail suspension, and forced swim. Sample sizes are indicated in each figure. All experiments were conducted according to the National Institute of 
Health guidelines for animal care and were approved by the Institutional Animal Care and Use Committees at University of lowa.

\section{Behavioral Procedures}

General: Mice were housed under regular light cycle lights on/off at 0900/2100 DST $\left(0800 / 2000\right.$ non-DST). The average ambient temperature was $22^{\circ} \mathrm{C}$ and mice were provided with food and water ad libitum. All experiments were conducted during the animals' light cycle. All equipment was cleaned between trials with $70 \%$ ethanol.

Open Field: Mice were placed in a $40 \mathrm{~cm}$ x $40 \mathrm{~cm}$ arena for 10 minutes under 115-130 lux. Activity was tracked by EthoVision software (Noldus, Leesburg, VA) and analyzed for total distance traveled and tendency to stay at edge of the arena. For the latter, the arena was divided into the periphery and the center where each comprised $50 \%$ of the total surface area of the arena.

Rotarod: Mice were placed on the rotating drum of an accelerating rotarod (UGO Basile or IITC Life Science Mouse), and the time to fall or second passive rotation was recorded for each mouse. The speed of the rotarod accelerated from 4 to $40 \mathrm{rpm}$ over a 5-minute period. Mice were given 3 trials/day for 5 days with a maximum time of 5 minutes, with at least a 10-minute inter-trial interval. Latency to fall or second passive rotation were recorded for each mouse each day.

Erasmus Ladder: Mice were subjected to the Erasmus Ladder task (Noldus, Wageningen, The Netherlands) which has been described in detail elsewhere (Van Der Giessen et al., 2008), with some modifications. Briefly, the mice were trained on the 
Erasmus Ladder for 42 trials per day for a total of 4 consecutive days. Trials were separated by a random inter-trial interval ranging from 11-20 seconds.

Tail suspension test: Mice were suspended approximately $46-48 \mathrm{~cm}$ from the tabletop by lab tape wrapped around their tails, which was then attached to a hook on a horizontal rod to avoid bending the tail. Mice were video recorded for 6 minutes. Mice were removed from the apparatus if they climbed their tails to the top of the horizontal rod; data from these animals was excluded from analysis.

Forced swim test: Mice were placed in clear acrylic cylinders (outer diameter: 23 $\mathrm{cm}$, inner diameter: $21.5 \mathrm{~cm}$, height: $34 \mathrm{~cm}$ ) filled halfway with water maintained at 20 $25^{\circ} \mathrm{C}$, and video recorded for 6 minutes. Trials were analyzed for latency to float and percentage of time immobile during the last 4 minutes of the trial.

Elevated zero maze: Mice were placed in a custom-built white plastic maze elevated $42.5 \mathrm{~cm}$ off the table with an internal diameter of $33.7 \mathrm{~cm}$ and outer diameter of $46 \mathrm{~cm}$ (internal pathway $5.8 \mathrm{~cm}$ wide). Walls on the closed sections were $10 \mathrm{~cm}$ high, and the lip on open sections was $0.6 \mathrm{~cm}$ high. Each mouse underwent a single 5-minute trial under 250 lux (open sections). Activity was tracked by EthoVision software for distance traveled, velocity, and duration spent in open/closed sections.

Three-chamber social preference test: Mice were placed in a matte, black plastic rectangular arena $(\mathrm{L} \times \mathrm{W} \times \mathrm{H}=51 \mathrm{~cm} \times 25.4 \mathrm{~cm} \times 25.4 \mathrm{~cm})$ divided into three compartments, with $10 \mathrm{~cm}$ wide opening between compartments and empty clear acrylic perforated cylinders in center of each outer compartment. Mice were habituated to the entire testing apparatus for 10 minutes. For the test, a novel conspecific mouse was placed under one cylinder while a novel object (colored plastic blocks) were placed 
under the other cylinder, and the test mouse was allowed to explore for 10 minutes. Mice were placed in middle compartment at the beginning of the test. Mice were removed from the apparatus if they climbed to the tops of the walls of the arena. Activity was tracked by EthoVision software for distance traveled, velocity, and interaction time (calculated based on when nose of animal was within $1.5 \mathrm{~cm}$ of cylinder but excluding instances where mouse was rearing or climbing on the cylinder).

Statistics

Data were analyzed with sexes combined and with sexes separated to assess for sex as a biological variable. Data were graphed and analyzed using GraphPad Prism 8.0 (GraphPad Software, San Diego, CA), and are graphically represented as mean \pm standard error of the mean (SEM) for each group. Data were analyzed using the statistical test noted in results and figure legends. Results were considered significant when $\mathrm{p}<0.05$ (denoted in all graphs as follows: * $\mathrm{p}<0.05 ;{ }^{* *} \mathrm{p}<0.01$ ).

Results

Mice lacking $\mathrm{Ca}_{v} 1.3$ display normal locomotor and exploratory behavior

We first sought to determine whether $\mathrm{Ca}_{v} 1$.3-deficient mice display abnormal locomotor and exploratory behaviors. In the open field task, we observed no differences in the distance traveled by $\mathrm{Ca}_{\mathrm{v}} 1.3 \mathrm{Hap}$ or $\mathrm{KO}$ mice compared to WT littermates (Fig. 1a), nor a difference in the mean velocity of exploration (Fig. 1b). We did observe a main effect of sex for distance traveled (Fig. S1a) and mean velocity (Fig. S1b); post- 
hoc testing revealed that this was driven by a difference between WT females and Cav1.3 KO males for both distance traveled and mean velocity (Tukey's posthoc test $p=0.011$ for both). When sexes were separated, we found no main effect of genotype for distance traveled or mean velocity (Fig. S1a-b). These data suggest that $\mathrm{Ca}$ 1.3 deficiency does not cause major impairments in baseline locomotion or exploration.

Mice lacking $\mathrm{Ca}_{v} 1.3$ display impaired motor performance

Although $\mathrm{Ca}_{v} 1.3$ does not appear essential for basic locomotor and exploratory function (Figs. 1 and $\mathrm{S} 1$ ), we hypothesized that $\mathrm{Ca}_{v} 1.3$ may be involved in learning related to cerebellar-mediated motor performance. Therefore, we explored the ability of mice lacking $\mathrm{Ca}_{v} 1.3$ to learn the accelerated rotarod task. $\mathrm{Ca}_{\mathrm{v}} 1.3 \mathrm{KO}$ mice displayed impaired motor performance compared to their WT and Hap littermates (Fig. 1c). $\mathrm{Ca}_{v} 1.3$ $\mathrm{KO}$ mice demonstrated the ability to learn the accelerating rotarod task itself, as evidenced by improvement in performance between days 1 and 5 (paired two-tailed $t$ test, $t(26)=4.62, p<0.0001)$. These data support the hypothesis that $\mathrm{Ca}_{\mathrm{v}} 1.3$ is relevant in motor learning.

Given the sex differences in the incidence of several disorders associated with $\mathrm{Ca}_{\mathrm{v}} 1.3$, such as autism and depression, we sought to determine whether sex differences were the primary drivers of the motor performance deficits we observed. When we analyzed rotarod data from male mice alone, we observed that there was a genotype by day interaction effect, but there was no main effect of genotype (Fig. S1c). In female mice, we observed a main effect of genotype, but post-hoc Tukey's multiple comparisons test identified no differences between genotypes (Fig. S1c). We conclude 
that the differences in rotarod performance are not likely to be driven primarily by sex differences.

$\mathrm{Ca}_{\mathrm{v}} 1.3$ knockout mice display associative learning deficits

The Erasmus Ladder task permits assessment of a variety of behaviors, including associative learning (ability to learn visual and sensory start cues), cerebellum-dependent associative learning (learning to time a jump for an auditory cue), and motor coordination (missteps). The task parameters have been described in detail elsewhere (Van Der Giessen et al., 2008). Briefly, in this task, mice start a motor trial after a cue (a bright light, followed by air if they do not begin within 3 seconds of the light coming on). The apparatus measures the animal's steps across a discontinuous ladder and detects errors for each of 42 trials in a session. We were not able to assess cerebellum-dependent tone-cued associative learning with this task because the conditioned stimulus is a tone and $\mathrm{Ca}_{\vee} 1.3 \mathrm{KO}$ mice are congenitally deaf. However, since $\mathrm{Ca}_{v} 1.3 \mathrm{KO}$ mice have normal vision and mechanosensation (Busquet et al., 2010; Clark et al., 2003), we were able to assess light- and air-cued associative learning and motor coordination. Most animals tended not to start the task following the light cue (Fig. 2a). However, WT and Hap mice generally started the task following the air cue, while Cav1.3 KO mice responded to this cue less frequently (Fig. 2b). Ca $1.3 \mathrm{KO}$ mice also started the trial before any cue more often (Fig. 2c), whereas WT and Hap mice learned quickly not to start a trial without either a light or air cue. These data support previous studies showing that $\mathrm{Ca}_{v} 1.3$ is important for associative learning. 
Interestingly, once on the ladder, Cav1.3 KO mice did not display an increase in missteps compared to WT or Hap mice, suggesting that loss of $\mathrm{Ca}_{v} 1.3$ does not cause ataxia or coordination problems (Fig. 2d). The normal motor performance of Cav1.3 KO mice on the motor aspects of the Erasmus Ladder suggests that the observed rotarod motor deficits are not secondary to incoordination.

Mice lacking $\mathrm{Ca}_{v} 1.3$ do not display depression-like or anxiety-like behaviors

Since genetic variation in Cav1.3 has been associated with mood disorders in humans (Ament et al., 2015; Cross-Disorder Group of the Psychiatric Genomics, 2013; Psychiatric, 2011) and antidepressant-like behavior in male mice (Busquet et al., 2010), we explored whether mice lacking Cav1.3 have abnormal depression-like or anxiety-like behaviors. In the tail suspension test, which has been used as a predictor of antidepressant efficacy (Castagne, Moser, \& Porsolt, 2009), we observed no differences in time spent immobile between genotypes (Fig. 3a). When sexes were separated, no differences between sexes or genotypes was observed (Fig. S2a). Similarly, in the forced swim test of behavioral despair (Castagne, Moser, et al., 2009; Castagne, Porsolt, \& Moser, 2009), we found no differences between genotypes in time spent immobile (Fig. 3b) or in latency to begin floating (Fig. 3c). When sexes were separated, we observed no differences between genotypes for time immobile (Fig. S2b) or latency to float (Fig. S2c).

We examined anxiety-like behaviors using the elevated zero maze and the open field task, both of which measure the animal's propensity to explore a riskier part of the environment. In the elevated zero maze, we found no differences in percent time spent 
in the open segments by genotype (Fig. 3d); the same was true when animals were separated by sex (Fig. S2d). In the open field task, time spent in the center of the field is associated with reduced anxiety, whereas time spent in the periphery (also called thigmotaxis) is associated with higher anxiety (Gershenfeld \& Paul, 1997; Treit \& Fundytus, 1988). In the open field test, we observed no differences in thigmotaxis with sexes combined (Fig. 3e) or separated (Fig. S2e).

$\mathrm{Ca}_{v} 1.3$ knockout mice display normal preference for social interaction

Since rare mutations in $\mathrm{Ca}_{v} 1.3$ have been linked to autism, we wanted to test whether loss of $\mathrm{Ca}_{v} 1.3$ would impair social preference. We tested this using the threechamber social preference test (Williams, Yee, Smith, Murphy, \& Umemori, 2016; Yang, Silverman, \& Crawley, 2011). We found that mice lacking one or both copies of $\mathrm{Ca}_{\mathrm{v}} 1.3$ prefer to explore animals rather than objects in the social preference test, similarly to WT littermates (Fig. 4a). Ca 1.3 Hap mice displayed a stronger preference for conspecifics over objects compared to WT and KO mice, while WT and KO mice were not different from each other. We found no genotype-specific differences in time spent exploring stimuli during the task (Fig. 4b) nor in movement velocity during exploration (Fig. 4c), supporting the open field and elevated zero maze data showing no differences in basal locomotor function or velocity (Fig. 1a-b and S3).

\section{Discussion}

L-type voltage-gated calcium channels (LVGCCs) have important roles in learning and memory and have been linked to multiple human neuropsychiatric 
diseases. Here we investigated the behavioral phenotypes caused by loss of one specific LVGCC, Cav1.3. We found that complete loss of Cav1.3 is associated with deficits in the accelerating rotarod task (Fig. 1c), indicative of impaired motor learning or performance. We also identified abnormalities in light- and air-cued associative learning on the Erasmus Ladder task in Cav1.3 KO mice (Fig. 2), which concurs with previous studies demonstrating the importance of Cav1.3 in associative learning. These data taken together with normal locomotor exploratory behavior (Figs. 4 and S3) and lack of ataxia or incoordination on Erasmus Ladder (Fig. 2) suggest that the rotarod deficits observed in Cav1.3 KO mice are not related to underlying deficits in locomotor ability, but rather, demonstrate a deficit in motor performance or learning.

Our data differ from previous studies of Cav1.3 KO mice which demonstrated normal rotarod performance (Clark et al., 2003; McKinney \& Murphy, 2006). This could be attributable to differences in task protocols or genetic background. We used an accelerating version of this task, whereas other studies have used a fixed speed apparatus (Clark et al., 2003) which ran at a slower rate (18 rpm) than our top speed (40 rpm), and may not have revealed the phenotype we observed. A previous study demonstrating normal accelerating rotarod performance used Cav1.3 KO mice on a C57BL/6:129Sve F2 hybrid background (McKinney \& Murphy, 2006) which may account for the difference in results. Additionally, we used larger sample sizes than either previous study, which may have provided more power to detect this motor learning or performance phenotype. Our results regarding normal locomotor activity and exploration agree with those of earlier studies (Busquet et al., 2010; Clark et al., 2003). 
We also did not observe depression-like behaviors in Cav1.3 $\mathrm{KO}$ mice as has been previously described (Busquet et al., 2010). Notably, other studies of this animal model have also not detected depression-like or anxiety-like behaviors (Marschallinger et al., 2015; McKinney \& Murphy, 2006). The origin of this discrepancy is unclear, and merits further investigation. As mentioned above, we used larger sample sizes as compared to these previous studies, which may partially explain some of the differences between our results and those of other groups.

Our Erasmus Ladder data support the hypothesis that Cav1.3 is important for associative learning when mildly aversive stimuli (bright light and air puffs) are used. These data are consistent with the findings of (McKinney \& Murphy, 2006) where Cav1.3 deletion disrupted consolidation but not extinction of contextual fear conditioning. Notably, when these mice are exposed to stronger fear conditioning stimuli there appear to be no differences in either consolidation or extinction of fear learning (Busquet, Hetzenauer, Sinnegger-Brauns, Striessnig, \& Singewald, 2008), suggesting that Cav1.3 is not essential for all forms of aversive learning but may modulate learning under less stressful circumstances. Conversely, one study suggests that activating Cav1.3 specifically in the ventral tegmental area contributes to cocaine preference while also inducing depressive-like behavior and social preference deficits (Martinez-Rivera et al., 2017). There is now also a mouse line which overexpresses Cav1.3 in excitatory forebrain neurons (Krueger et al., 2017) which might reveal whether Cav1.3 in specific brain regions or neuronal subtypes is associated with specific types of behavioral circuit dysfunction. For example, it would be interesting to study whether Cav1.3 plays a role in 
milder forms of reward learning, such as operant conditioning paradigms, as different forms of learning may be mediated by different neural circuits.

Summary

In summary, our data demonstrate the importance of Cav1.3 in both motor and associative learning. Abnormalities in motor and associative learning have been demonstrated in multiple neuropsychiatric disorders, including autism, bipolar disorder, and schizophrenia. Interestingly, despite the association of Cav1.3 with mood disorders in humans, we do not find evidence of affective or anxiety-like abnormalities in mice lacking Cav1.3. This suggests that perhaps Cav1.3 contributes to common cognitive deficits across neuropsychiatric disorders. Therefore, therapies that aim to modulate Cav1.3 function may have broad applicability across neuropsychiatric conditions.

Author Contributions: All authors contributed to the study conception and design. Material preparation, data collection and analysis were performed by Marisol Lauffer, Hsiang Wen, Bryn Myers, Ashley Plumb, and Aislinn Williams. The first draft of the manuscript was written by Aislinn Williams and all authors commented on previous versions of the manuscript. All authors read and approved the final manuscript.

Acknowledgements: The authors would like to thank Shane Heiney and the Neural Circuits and Behavior Core at the University of lowa, Dr. Kumar Narayanan, and Parker Abbott for training and behavioral equipment used in this study. We also thank Dr. 
Geoffrey Murphy for Cav1.3 knockout mice, and the members of the lowa Neuroscience Institute for thoughtful comments on this manuscript.

Funding: This project was funded by NIH R01MH118240 (K.L.P.), K01MH106824

(K.L.P.), KL2TR002536 (A.J.W.), the Roy J. Carver Charitable Trust (A.J.W.), the Graduate Program in Neuroscience (A.P.), and the Roy J. and Lucille A. Carver College of Medicine (B.M.).

Conflict of interest statement: The authors declare that they have no conflicts of interest.

\section{References}

Ament, S. A., Szelinger, S., Glusman, G., Ashworth, J., Hou, L., Akula, N., . . Roach, J. C. (2015). Rare variants in neuronal excitability genes influence risk for bipolar disorder. Proc Natl Acad Sci U S A, 112(11), 3576-3581.

doi:10.1073/pnas.1424958112

Brandt, A., Striessnig, J., \& Moser, T. (2003). CaV1.3 channels are essential for development and presynaptic activity of cochlear inner hair cells. J Neurosci, 23(34), 10832-10840. Retrieved from https://www.ncbi.nlm.nih.gov/pubmed/14645476

Busquet, P., Hetzenauer, A., Sinnegger-Brauns, M. J., Striessnig, J., \& Singewald, N. (2008). Role of L-type Ca2+ channel isoforms in the extinction of conditioned fear. Learn Mem, 15(5), 378-386. doi:10.1101//m.886208 
Busquet, P., Nguyen, N. K., Schmid, E., Tanimoto, N., Seeliger, M. W., Ben-Yosef, T., . . Singewald, N. (2010). CaV1.3 L-type Ca2+ channels modulate depression-like behaviour in mice independent of deaf phenotype. Int J Neuropsychopharmacol, 13(4), 499-513. doi:10.1017/S1461145709990368

Castagne, V., Moser, P., \& Porsolt, R. D. (2009). Behavioral Assessment of Antidepressant Activity in Rodents. In nd \& J. J. Buccafusco (Eds.), Methods of Behavior Analysis in Neuroscience. Boca Raton (FL).

Castagne, V., Porsolt, R. D., \& Moser, P. (2009). Use of latency to immobility improves detection of antidepressant-like activity in the behavioral despair test in the mouse. Eur J Pharmacol, 616(1-3), 128-133. doi:10.1016/j.ejphar.2009.06.018

Catterall, W. A., Perez-Reyes, E., Snutch, T. P., \& Striessnig, J. (2005). International Union of Pharmacology. XLVIII. Nomenclature and structure-function relationships of voltage-gated calcium channels. Pharmacol Rev, 57(4), 411-425. doi:10.1124/pr.57.4.5

Clark, N. C., Nagano, N., Kuenzi, F. M., Jarolimek, W., Huber, I., Walter, D., . . . Seabrook, G. R. (2003). Neurological phenotype and synaptic function in mice lacking the CaV1.3 alpha subunit of neuronal L-type voltage-dependent Ca2+ channels. Neuroscience, 120(2), 435-442. doi:10.1016/s0306-4522(03)00329-4

Cross-Disorder Group of the Psychiatric Genomics, C. (2013). Identification of risk loci with shared effects on five major psychiatric disorders: a genome-wide analysis. Lancet, 381(9875), 1371-1379. doi:10.1016/S0140-6736(12)62129-1

Gershenfeld, H. K., \& Paul, S. M. (1997). Mapping quantitative trait loci for fear-like behaviors in mice. Genomics, 46(1), 1-8. doi:10.1006/geno.1997.5002 
Hell, J. W., Westenbroek, R. E., Warner, C., Ahlijanian, M. K., Prystay, W., Gilbert, M. M., . . Catterall, W. A. (1993). Identification and differential subcellular localization of the neuronal class $C$ and class D L-type calcium channel alpha 1 subunits. J Cell Biol, 123(4), 949-962. doi:10.1083/jcb.123.4.949

Kim, S. H., Park, Y. R., Lee, B., Choi, B., Kim, H., \& Kim, C. H. (2017). Reduction of Cav1.3 channels in dorsal hippocampus impairs the development of dentate gyrus newborn neurons and hippocampal-dependent memory tasks. PLoS One, 12(7), e0181138. doi:10.1371/journal.pone.0181138

Krueger, J. N., Moore, S. J., Parent, R., McKinney, B. C., Lee, A., \& Murphy, G. G. (2017). A novel mouse model of the aged brain: Over-expression of the L-type voltage-gated calcium channel CaV1.3. Behav Brain Res, 322(Pt B), 241-249. doi:10.1016/j.bbr.2016.06.054

Marschallinger, J., Sah, A., Schmuckermair, C., Unger, M., Rotheneichner, P., Kharitonova, M., ... Aigner, L. (2015). The L-type calcium channel Cav1.3 is required for proper hippocampal neurogenesis and cognitive functions. Cell Calcium, 58(6), 606-616. doi:10.1016/j.ceca.2015.09.007

Martinez-Rivera, A., Hao, J., Tropea, T. F., Giordano, T. P., Kosovsky, M., Rice, R. C., . .. Rajadhyaksha, A. M. (2017). Enhancing VTA Cav1.3 L-type Ca(2+) channel activity promotes cocaine and mood-related behaviors via overlapping AMPA receptor mechanisms in the nucleus accumbens. Mol Psychiatry, 22(12), 17351745. doi:10.1038/mp.2017.9 
McKinney, B. C., \& Murphy, G. G. (2006). The L-Type voltage-gated calcium channel Cav1.3 mediates consolidation, but not extinction, of contextually conditioned fear in mice. Learn Mem, 13(5), 584-589. doi:10.1101/lm.279006

Pinggera, A., Lieb, A., Benedetti, B., Lampert, M., Monteleone, S., Liedl, K. R., . . . Striessnig, J. (2015). CACNA1D de novo mutations in autism spectrum disorders activate Cav1.3 L-type calcium channels. Biol Psychiatry, 77(9), 816-822. doi:10.1016/j.biopsych.2014.11.020

Platzer, J., Engel, J., Schrott-Fischer, A., Stephan, K., Bova, S., Chen, H., . . . Striessnig, J. (2000). Congenital deafness and sinoatrial node dysfunction in mice lacking class D L-type Ca2+ channels. Cell, 102(1), 89-97. doi:10.1016/s0092-8674(00)00013-1

Psychiatric, G. C. B. D. W. G. (2011). Large-scale genome-wide association analysis of bipolar disorder identifies a new susceptibility locus near ODZ4. Nat Genet, 43(10), 977-983. doi:10.1038/ng.943

Scholl, U. I., Goh, G., Stolting, G., de Oliveira, R. C., Choi, M., Overton, J. D., . . Lifton, R. P. (2013). Somatic and germline CACNA1D calcium channel mutations in aldosterone-producing adenomas and primary aldosteronism. Nat Genet, 45(9), 1050-1054. doi:10.1038/ng.2695

Shi, L., Chang, J. Y., Yu, F., Ko, M. L., \& Ko, G. Y. (2017). The Contribution of L-Type Cav1.3 Channels to Retinal Light Responses. Front Mol Neurosci, 10, 394. doi:10.3389/fnmol.2017.00394

Treit, D., \& Fundytus, M. (1988). Thigmotaxis as a test for anxiolytic activity in rats. Pharmacol Biochem Behav, 31(4), 959-962. doi:10.1016/0091-3057(88)90413-3 
Van Der Giessen, R. S., Koekkoek, S. K., van Dorp, S., De Gruijl, J. R., Cupido, A., Khosrovani, S., .. . De Zeeuw, C. I. (2008). Role of olivary electrical coupling in cerebellar motor learning. Neuron, 58(4), 599-612.

doi:10.1016/j.neuron.2008.03.016

Williams, A. J., Yee, P., Smith, M. C., Murphy, G. G., \& Umemori, H. (2016). Deletion of fibroblast growth factor 22 (FGF22) causes a depression-like phenotype in adult mice. Behav Brain Res, 307, 11-17. doi:10.1016/j.bbr.2016.03.047

Yang, M., Silverman, J. L., \& Crawley, J. N. (2011). Automated three-chambered social approach task for mice. Curr Protoc Neurosci, Chapter 8, Unit 826. doi:10.1002/0471142301.ns0826s56 
Figure Captions

Fig. 1 Cav1.3 KO mice have normal locomotor exploration but impaired motor learning.

a No differences observed in open field distance (one-way ANOVA, $F_{2,45}=2.81, p=0.07$ ).

b No differences in mean velocity of movement in open field (one-way ANOVA,

$\left.\mathrm{F}_{2,45}=2.81, \mathrm{p}=0.07\right)$. $\mathrm{c} \mathrm{KO}$ mice perform significantly worse on the accelerating rotarod

test of motor learning compared to WT mice. There is a main effect of genotype (two-

way repeated-measures $\mathrm{ANOVA}, \mathrm{F}_{2,87}=3.37, \mathrm{p}=0.04$ ) as well as a main effect of

training for all groups (two-way repeated-measures ANOVA, $F_{2.514,218.7}=84.7$,

$p<0.0001)$. There is also a genotype $x$ training interaction effect (two-way repeated-

measures ANOVA, $\left.\mathrm{F}_{8,348}=2.39, \mathrm{p}=0.02\right)$. Hap mice are not distinguishable from WT or KO.

Fig. 2 Cav1.3 KO mice display impaired associative learning on the Erasmus Ladder task. a All mice demonstrate low propensity to leave the start box on the light cue; no differences noted between genotypes. b KO mice are significantly less likely to leave the start box with the air cue compared to WT and Hap littermates (main effect of genotype, two-way repeated measures ANOVA, $F_{2,39}=58.07, p<0.01$; both WT and Hap mice differed significantly from KO by Tukey's posthoc multiple comparisons test $\mathrm{p}<0.01)$. No difference between WT and Hap mice. c KO mice are significantly more likely to move onto the ladder prior to any start cue compared to WT and Hap littermates (two-way repeated-measures ANOVA, main effect of genotype, $F_{2,39}=58.9$, 
$p<0.01$; Tukey's multiple comparisons test $p<0.01$ ). No difference between WT and Hap mice. d All animals have a reduction in missteps over time (two-way repeated-measures ANOVA, main effect of genotype, $\left.F_{2,39}=0.76, p=0.48\right)$. There is a significant genotype by day interaction effect (two-way repeated-measures ANOVA, genotype $x$ training interaction effect, $\left.F_{6,117}=2.84, p<0.05\right)$, but Tukey's HSD identified no differences between genotypes on any individual day.

Fig. 3 Cav1.3 KO mice have no deficits in affective or anxiety-like behaviors. a KO mice are not different from WT and Hap littermates in time spent in escape behavior on the tail suspension test (one-way ANOVA, $F_{2,85}=0.33, p=0.72$ ). KO mice are not different from WT and Hap littermates in the forced swim test in terms of either percent time spent immobile (b) (one-way ANOVA, $\mathrm{F}_{2,87}=1.63, \mathrm{p}=0.20$ ) or latency to start floating (c) (one-way ANOVA, $\mathrm{F}_{2,81}=0.77, \mathrm{p}=0.46$ ). $\mathrm{KO}$ mice have normal anxiety-like behaviors in both the elevated zero maze measured as time spent in closed segments (d) (one-way ANOVA, $F_{2,45}=0.65, p=0.53$ ) and time spent in the periphery of the open field test $(\mathbf{e})$ (one-way ANOVA, main effect of genotype, $\mathrm{F}_{2,45}=0.21, \mathrm{p}=0.81$ ).

Fig. 4 Cav1.3 KO mice do not display social interaction deficits. a KO mice display normal preference for social interaction with a novel mouse over a novel object (twoway ANOVA, main effect of test object, $F_{1,86}=47.19, p<0.01$ ). Hap mice spend more time with a novel mouse than their WT and KO littermates (two-way ANOVA, main effect of genotype, $F_{2,86}=5.80, p<0.01$, Tukey's HSD $\left.p<0.05\right)$, but the same amount of time exploring a novel object as their WT and KO littermates. There was no interaction effect 
$\left(F_{2,86}=1.56, p=0.22\right)$. There are no differences between genotypes in total exploration during the social interaction task (b) (one-way ANOVA, $F_{2,44}=0.46, p=0.63$ ) or velocity of movements during the task (c) (one-way ANOVA, $F_{2,44}=0.47, p=0.63$ ).

Fig. S1 Data from Figure 1 separated by sex. Overall, female mice cover statistically more distance (a) (two-way ANOVA, $\mathrm{F}_{1,42}=7.96, \mathrm{p}=0.007$ ) at a greater speed (b) (twoway ANOVA, $F_{1,42}=7.96, p=0.007$ ) than male mice in the open field task. There is no main effect of genotype for either distance (two-way ANOVA, $F_{2,42}=3.08, p=0.057$ ) or velocity (two-way ANOVA, $\mathrm{F}_{2,42}=3.08, \mathrm{p}=0.057$ ). $\mathrm{c}$ For the rotarod task, there is a genotype by day interaction effect in males (interaction effect, two-way repeatedmeasures ANOVA, $\mathrm{F}_{8,164}=2.02, \mathrm{p}=0.047$ ) but no main effect of genotype (two-way repeated-measures ANOVA, $F_{2,41}=2.51, p=0.09$ ). For females on the rotarod task, both WT and Hap mice are different from KO, but WT and Hap females are not significantly different (two-way repeated-measures ANOVA, $F_{2,43}=4.05, p=0.025$; Tukey's HSD found no significant differences between groups).

Fig. S2 Data from Figure 3 separated by sex. There are no sex or genotype effects for any affective or anxiety-like task. a Tail suspension test: two-way ANOVA, main effect of genotype $F_{2,82}=0.34, p=0.71$; main effect of sex, $F_{1,82}=0.001, p=0.97$; interaction effect $F_{2,82}=1.36, p=0.26$. $\mathbf{b}$ Forced swim test, immobility: two-way ANOVA, main effect of genotype $F_{2,84}=1.56, p=0.22$; main effect of sex, $F_{1,84}=0.012, p=0.91$; interaction effect $F_{2,82}=0.31, p=0.73$. c Forced swim test, latency to float: two-way ANOVA, main effect of genotype $F_{2,78}=0.96, p=0.39$; main effect of sex, $F_{1,78}=2.13, p=0.15$; interaction 
effect $F_{2,78}=0.84, p=0.43$. $\mathbf{d}$ Elevated zero maze, time in closed segments: two-way ANOVA, main effect of genotype, $F_{2,42}=0.39, p=0.53$. e Open field test, proportion of time in outer edge of arena: two-way ANOVA, main effect of genotype, $F_{2,42}=0.23$, $\mathrm{p}=0.79$.

Fig. S3 Additional data from the elevated zero maze. KO mice are not different from WT and Hap littermates in terms of distance traveled (a) (one-way ANOVA, $F_{2,45}=2.50$, $p=0.09$ ) or velocity of movement (b) (one-way ANOVA, $F_{2,45}=2.47, p=0.10$ ) on the elevated zero maze. When mice are separated by sex, there is no main effect of sex on elevated zero maze distance (c) (two-way ANOVA, main effect of genotype $F_{2,42}=2.36$, $p=0.11$; main effect of sex, $F_{1,42}=0.44, p=0.51$; interaction effect $F_{2,42}=0.70, p=0.50$ ) or velocity (d) (two-way ANOVA, main effect of genotype $F_{2,42}=2.34, p=0.11$; main effect of sex, $F_{1,42}=0.43, p=0.52$; interaction effect $\left.F_{2,42}=0.70, p=0.50\right)$. 

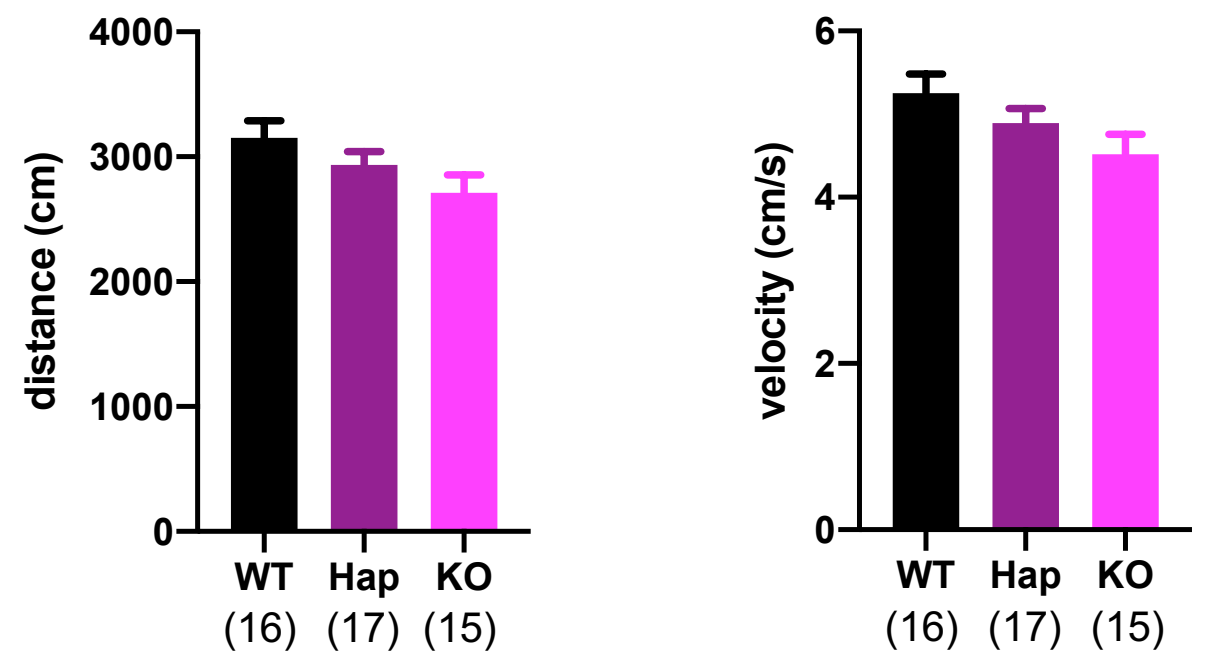

C Rotarod

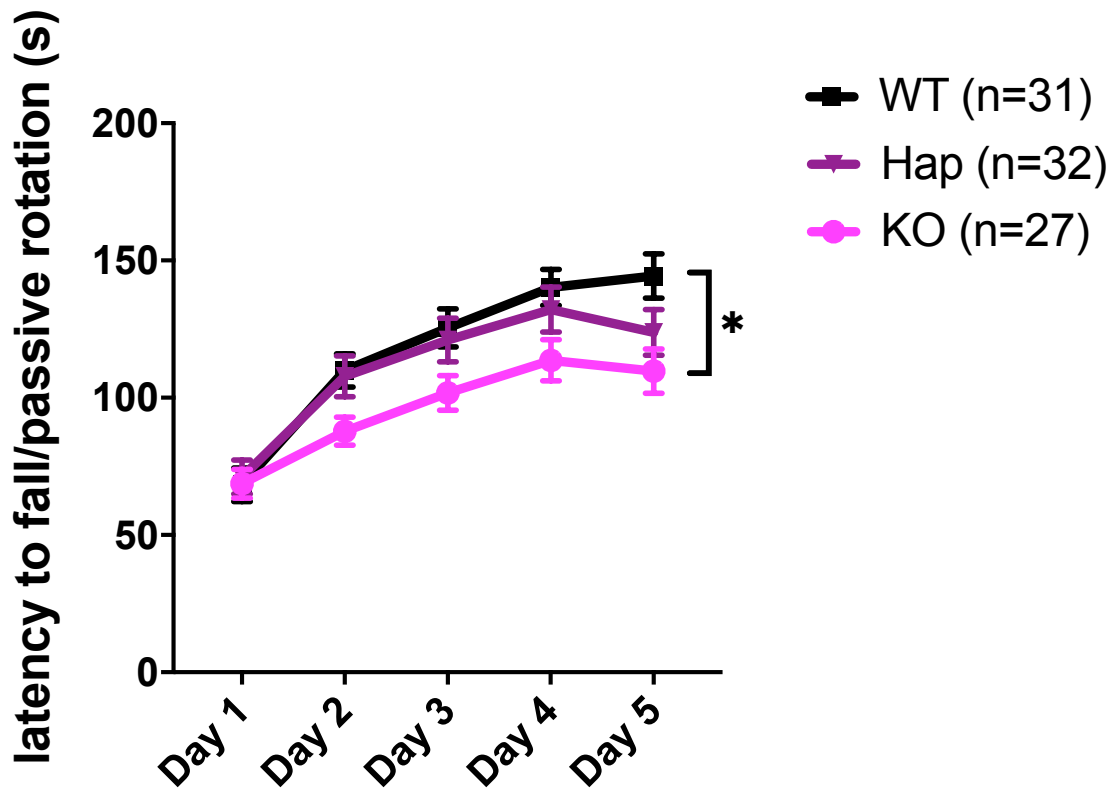


Leaving On Air Cue

$\rightarrow$ WT $(n=15)$

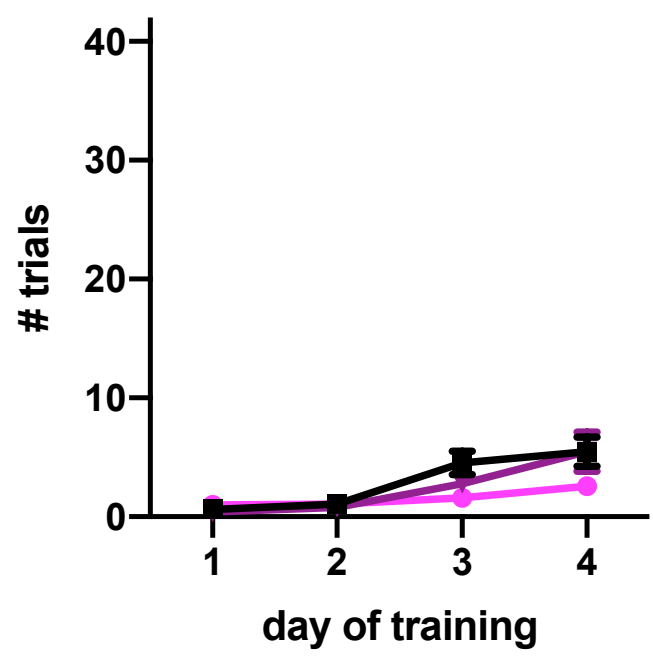

C
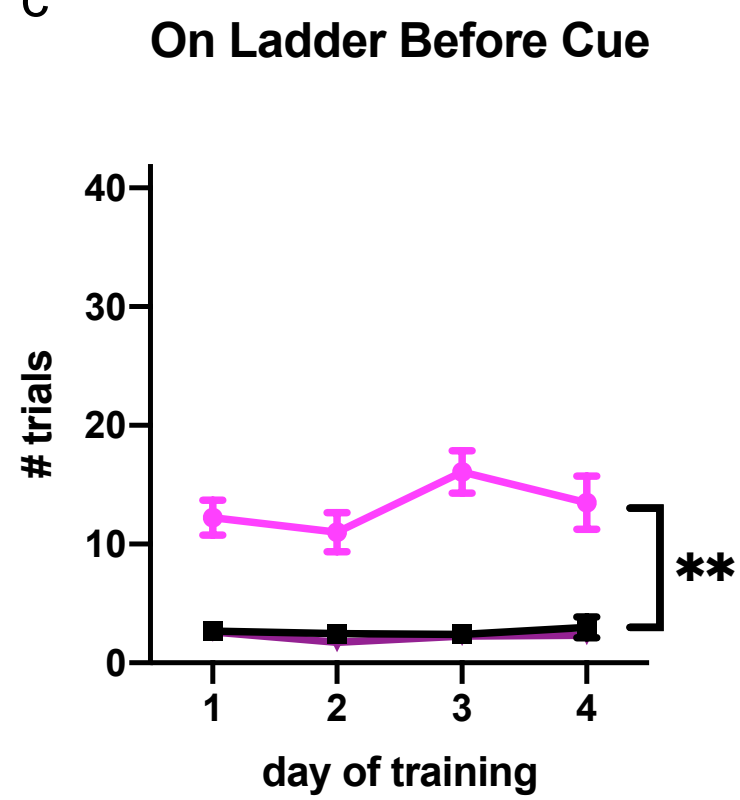

b $\rightarrow \operatorname{Hap}(n=15)$

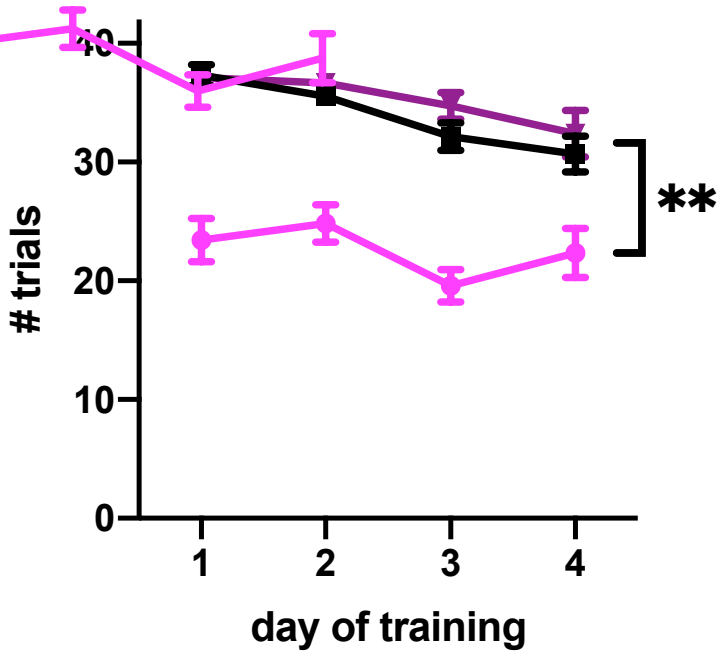

d

Missteps

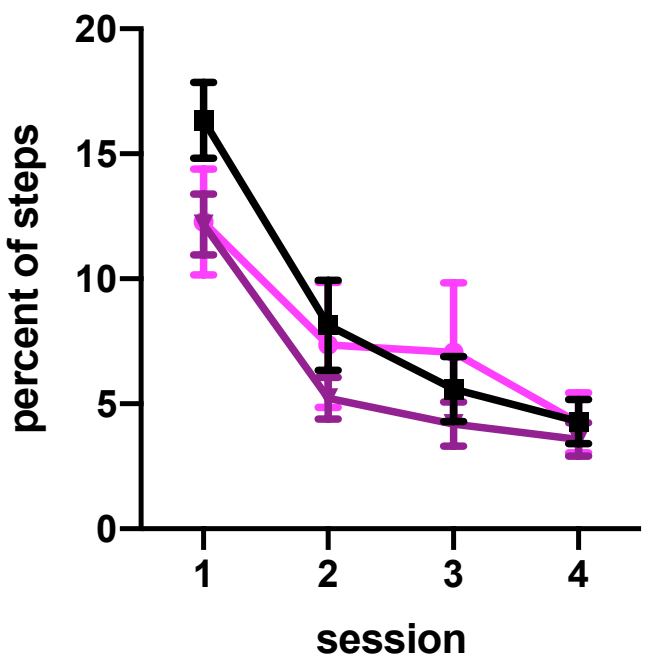


a Tail Suspension Test

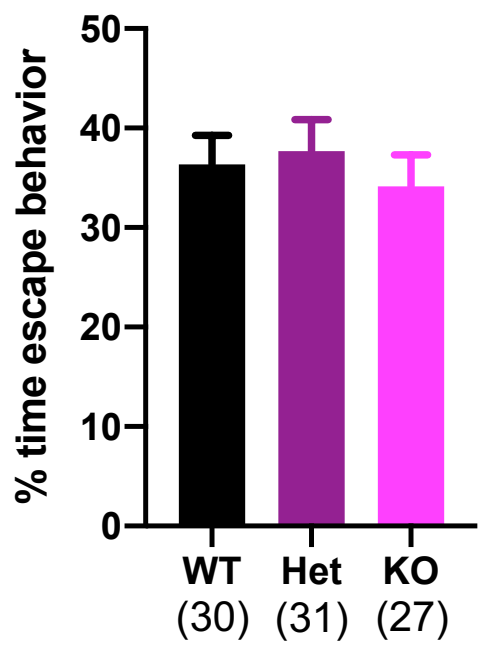

d

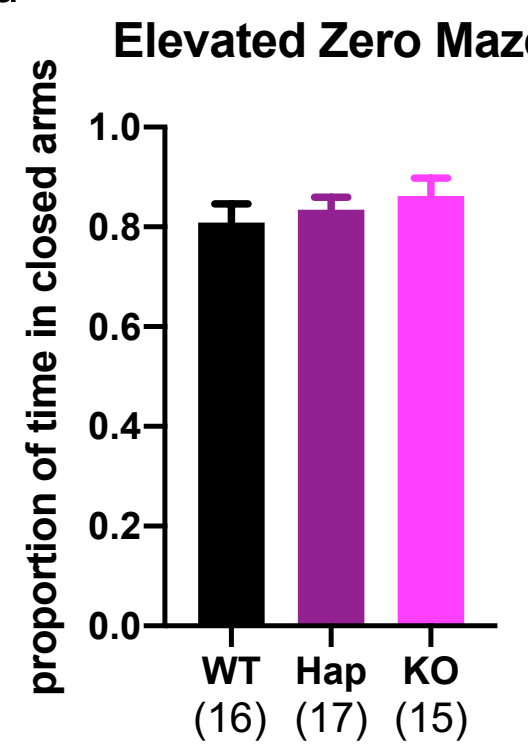

$\mathrm{b}$

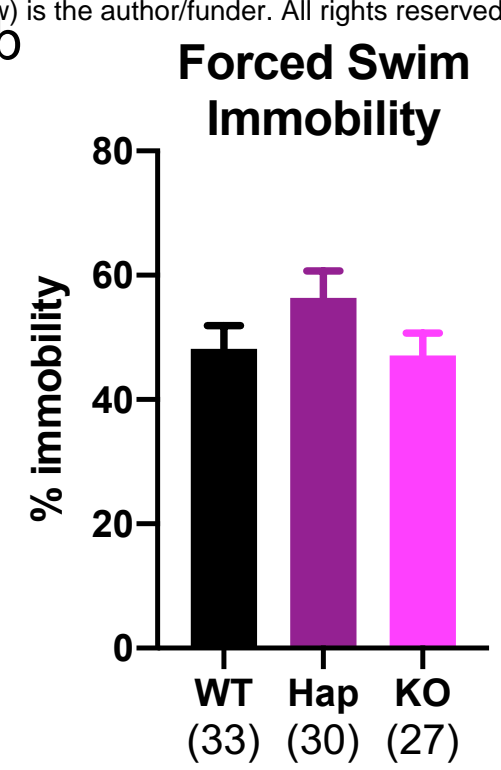

C

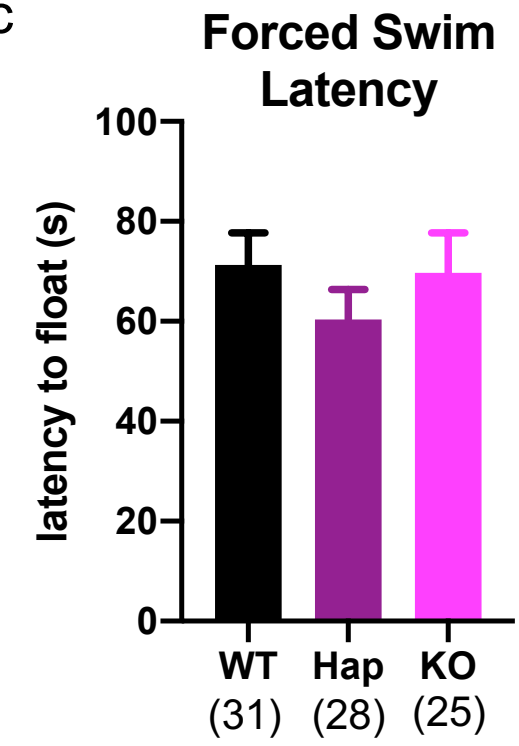

e

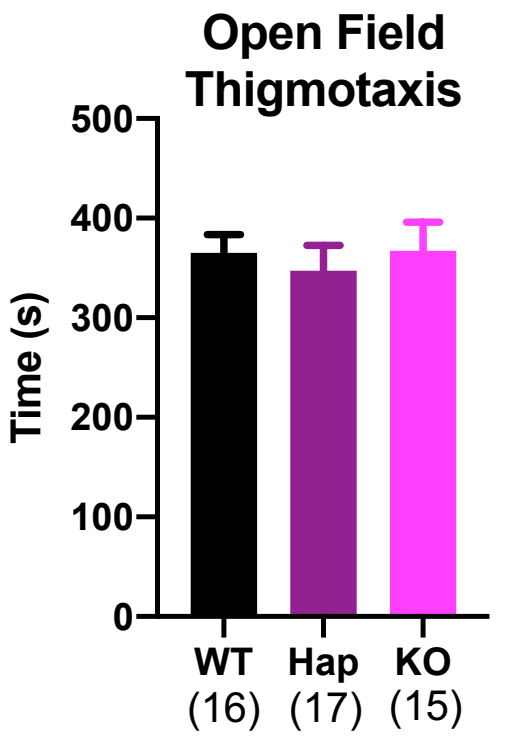


a

\section{Social Preference}

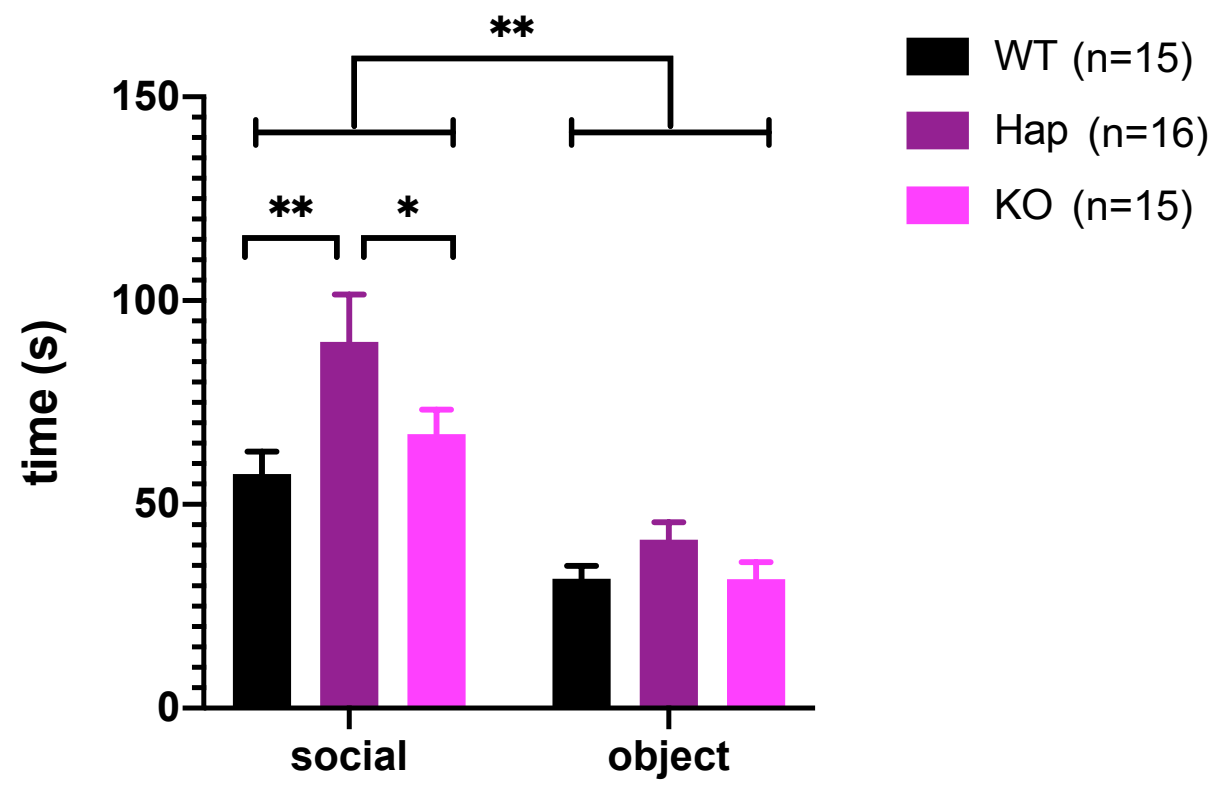

b

Exploration During Task

C Velocity
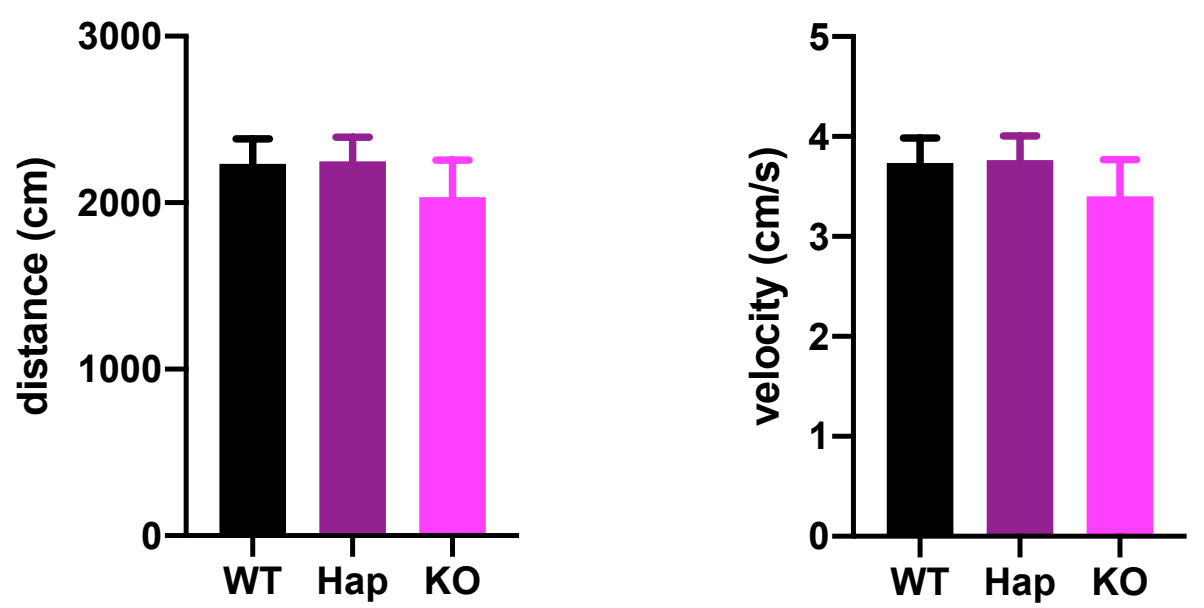